\title{
Model-based Gas Leakage Detection and Isolation in a Pressurized System via Laguerre Spectrum Analysis
}

\author{
Andreas Johansson, Wolfgang Birk, and Alexander Medvedev \\ Control Engineering Group \\ Luleå University of Technology \\ SE - 97187 Luleå, Sweden \\ Email: Andreas.Johansson, Wolfgang.Birk, Alexander.Medvedev@sm.luth.se
}

\begin{abstract}
This paper deals with gas-leakage detection and isolation in a fine coal injection vessel for a blast furnace process. Functions describing the expected time-domain behaviour of different leakages are developed, thus reducing the leakage isolation problem to pattern recognition. In order to lower the computational burden while preserving the essential dynamic behaviour of a leakage, a truncated Laguerre series representation is used. The Generalized Likelihood Ratio method is employed to compare the Laguerre spectrum of the residual with the Laguerre spectra of the predefined typical leakage functions. Tests of the developed algorithm have been successfully performed at SSAB Tunnplåt, Luleå.
\end{abstract}

\section{Introduction}

Pattern recognition is a general problem in $e . g$. signal processing, identification and fault detection, of which the latter is the topic of this article. Being able to single out the most probable cause of a non-zero residual by comparing it with a number of possible fault signatures is the core of fault isolation. It is then desirable to represent the essential dynamics of a signal compactly while removing irrelevant behaviour and noise. One way of doing this is to employ an orthonormal functional basis. This paper is an example of the use of Laguerre functions.

The application considered is a pulverized coal injection plant for a blast furnace process. Coal powder is very inflammable and can self-ignite in contact with air. This motivates the development of a leakage detection system which can identify a faulty valve and thus facilitate maintenance and prevent accidents. Efficient maintenance is very important since a continuous coal powder production is crucial to blast furnace operation. A sudden loss of coal injection capacity may lead to chilled hearth, a condition that can have serious consequences for the blast furnace. High performance pressure and flow control of the coal injection plant is reported in [1].

The basic terminology and techniques for fault detection can be found in the survey by Frank [2]. State estimation by linear and nonlinear observers is often used. Regarding linear systems, a number of different techniques exists, for example Unknown Input Observers, Dedicated Observers, Parity Space and Kalman Filter Methods. A survey on non-linear observers can be found in [3]. Fault detection in non-linear systems is for example treated in [4] and [5].

Faults can be divided in two groups: abrupt and incipient faults. Incipient faults are often treated by identification techniques [2], though, in this paper, an observerbased method is utilised, mostly due to the availability of reasonably good process models.

The paper's outline is as follows. First, a brief description of the coal injection plant is given. Then, a Hammerstein physical model and an observer-based gas leakage detection technique is presented. The residual of the observer is sensitive to particular types of faults in worn-out valves and is compared to the expected behaviour of a number of leakages. The residual and the leakage functions are represented by means of their corresponding Laguerre spectra. Finally, the results of real time experiments performed at SSAB Tunnplåt, Luleå are reported.

\section{Process description}

Fig. 1 shows the principal structure of a coal injection vessel.

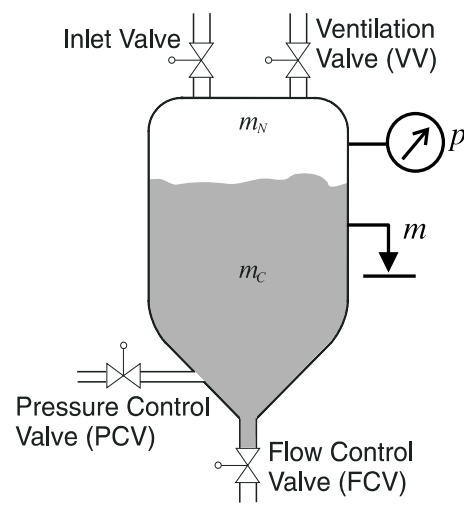

Figure 1: Schematic drawing of an injection vessel

One vessel is depressurized, charged and pressurized while the other vessel is injecting coal powder. In this manner, continuous coal powder flow into the blast furnace is achieved.

In Table 1, the nomenclature used in the sequel to 
Table 1: Process phases

\begin{tabular}{|l|l|}
\hline Phase & Description \\
\hline \hline Charging & $\begin{array}{l}\text { The pressure-less vessel is filled with } \\
\text { coal powder }\end{array}$ \\
\hline Pressurization & $\begin{array}{l}\text { The injection vessel is set under } \\
\text { pressure }\end{array}$ \\
\hline $\begin{array}{l}\text { Pressure hold- } \\
\text { ing }\end{array}$ & $\begin{array}{l}\text { Standby until the other vessel has } \\
\text { finished injection }\end{array}$ \\
\hline Injection & $\begin{array}{l}\text { The coal powder is injected into the } \\
\text { blast furnace }\end{array}$ \\
\hline Ventilation & $\begin{array}{l}\text { Depressurization and ventilation of } \\
\text { the vessel }\end{array}$ \\
\hline
\end{tabular}

Table 2: Leakages

\begin{tabular}{|l|l|c|}
\hline Leakage & $\begin{array}{l}\text { Possible } \\
\text { consequence }\end{array}$ & Notation \\
\hline \hline To the atmosphere & Loss of nitrogen & $\mathcal{A}$ \\
\hline $\begin{array}{l}\text { From the nitrogen } \\
\text { net }\end{array}$ & $\begin{array}{l}\text { Over-pressurized } \\
\text { vessel }\end{array}$ & $\mathcal{N}$ \\
\hline $\begin{array}{l}\text { To/from the injec- } \\
\text { tion pipe }\end{array}$ & Fire & $\mathcal{I}$ \\
\hline No Leakage & - & $\emptyset$ \\
\hline
\end{tabular}

refer to different process phases is summarized.

In order to be able to distinguish leakages in different valves, a process model for the pressurization and injection phases as well as a model for the ventilation phase are developed [6]. These models are based on physical principles and therefore nonlinear. The plant dynamics can be represented by

$$
\begin{aligned}
x(n+1) & =A x(n)+B g(u(n)) \\
y(n) & =h(x(n))
\end{aligned}
$$

where $A$ and $B$ are matrices containing identified values and the function $g$ is a non-linear transformation of the input signals. The state vector $x=\left[\begin{array}{ll}m_{C} & m_{N}\end{array}\right]^{T}$ represents the masses of coal and nitrogen in the vessel and the output vector $y=\left[\begin{array}{ll}m & p\end{array}\right]^{T}$ is a nonlinear transformation of $x$. The transformation, which is physically motivated, is uniquely invertible and thus $m_{C}$ and $m_{N}$ can be considered measurable.

\section{Fault detection}

The faults considered are three different leakages (Table 2).

\subsection{Leakage model}

The set of leakages is denoted $\mathcal{L} \triangleq\{\mathcal{A}, \mathcal{N}, \mathcal{I}, \emptyset\}$. A leakage can be interpreted as the flow through a valve with an unknown control signal. The nitrogen leakage flow can thus be represented by

$$
q_{\ell}=k_{\ell} f_{\ell}(\cdot) \quad \ell \in \mathcal{L}
$$

where $k_{\ell}$ is an unknown time-varying factor and $f_{\ell}(\cdot)$ is a function of the pressures on each side of the leakage. The trivial leakage function for the event of 'No Leakage' is $f_{\emptyset}=0$. The other leakage functions $\left(f_{\mathcal{A}}, f_{\mathcal{N}}\right.$ and $\left.f_{\mathcal{I}}\right)$ are developed from physical equations.

\subsection{Leakage detection}

The leakage flow is modeled as an extra term added to the right hand side of (1). The purpose of the fault detection algorithm is thus to calculate this term. However, the presence of noise makes it necessary to utilise feedback (i.e. observers) in order to obtain filtered estimates of the leakage flow. The nonlinear models developed in the modeling section are of the Hammerstein type and thus linear filtering can be used.

A linear observer for the system (1) is given by

$$
\hat{x}(n+1)=A \hat{x}(n)+B g(u(n))+K \epsilon(n)
$$

where

$$
\begin{aligned}
\epsilon(n) \triangleq\left[\epsilon_{C}(n) \quad \epsilon_{N}(n)\right. & ]^{T} \\
& \triangleq x(n)-\hat{x}(n) \\
& =h^{-1}(y(n))-\hat{x}(n)
\end{aligned}
$$

Since the state variables are decoupled (i.e. $A$ is diagonal), it is assumed that a diagonal observer gain matrix is sufficient. With the definitions above, it can be shown that the residual $\epsilon_{N}$ is the net leakage $q_{L}$, filtered through a first order filter, thus $\epsilon_{N}(n)=H(\mathbf{q}) q_{L}(n)$ where $\mathbf{q}$ is the forward shift operator. If the net leakage is assumed to be slowly varying in time, the residual divided by the static gain of the filter above is a good approximation of the net leakage whose measurements are corrupted by noise.

$$
\hat{q}_{L}(n)=\frac{1}{H(1)} \epsilon_{N}(n)
$$

\section{Leakage isolation}

The calculated leakage is assumed to be the sum of a scaled leakage function and a disturbance, i.e. $\hat{q}_{L}(n)=$ $k_{\ell} f_{\ell}(n)+e(n)$ where $\ell \in \mathcal{L}$ and the term $e(n)$ is stationary zero-mean white Gaussian noise with variance $\sigma^{2}$, i.e. $e(n) \in \mathcal{N}(0, \sigma)$

Actually, $e(n)$ is not Gaussian (probably due to unmodelled non-linearities), but this fact does not have any major influence. When the transient behavior is excluded from the data, the residual is fairly near normal distribution, but the results are virtually the same.

The factor $k_{\ell}$ in (2) is a measure of the size of the hole through which the leakage flow takes place. This means that $k_{\ell}$ varies slowly in time when describing incipient leakages. If it is assumed to be constant during a reasonably long period of time (for example a process cycle), it can be estimated using the Generalized Likelihood Ratio.

\subsection{Leakage hypothesis testing}

Four hypotheses $\left(\mathcal{H}_{\emptyset}, \mathcal{H}_{\mathcal{A}}, \mathcal{H}_{\mathcal{N}}\right.$ and $\left.\mathcal{H}_{\mathcal{I}}\right)$ are formed in agreement with the leakage events. The three leakage hypotheses are tested one by one against $\mathcal{H}_{\emptyset}$ using the Generalized Likelihood Ratio (GLR). If $\mathcal{H}_{\emptyset}$ is rejected in more than one of these tests, the hypothesis with the 
highest GLR is accepted. The likelihood functions for the hypotheses can be expressed as

$$
P_{\ell}\left(\hat{q}_{L}\right)=\prod_{n=1}^{N} \frac{1}{\sigma} \varphi\left(\frac{\hat{q}_{L}(n)-k_{\ell} f_{\ell}(n)}{\sigma}\right)
$$

where $\varphi$ is the scalar normal distribution density function with zero mean and unit variance. The GLR for each leakage hypothesis is

$$
\Lambda_{\ell}\left(\hat{q}_{L}\right)=\frac{\sup _{k_{\ell}>0} P_{\ell}\left(\hat{q}_{L}\right)}{P_{\emptyset}\left(\hat{q}_{L}\right)}
$$

The restriction on $k_{\ell}$ comes from the fact that a negative $k_{\ell}$ would imply a leakage flow from a lower pressure to a higher. It can be shown that under the conditions above, the leakage likelihood function, $P_{\ell}\left(\hat{q}_{L}\right)$, is maximized by

$$
\hat{k}_{\ell}=\arg \sup _{k_{\ell}>0} P_{\ell}\left(\hat{q}_{L}\right)=\max \left(0, \frac{\left\langle\hat{q}_{L}, f_{\ell}\right\rangle}{\left\langle f_{\ell}, f_{\ell}\right\rangle}\right)
$$

where $\langle\cdot, \cdot\rangle$ denotes scalar product in $R^{N}$, i.e.

$$
\left\langle f_{1}, f_{2}\right\rangle=\sum_{n=0}^{N-1} f_{1}(n) f_{2}(n)
$$

where $N$ is the time interval of the logged data set. The logarithmic GLR can then be expressed as

$$
\ln \left(\Lambda_{\ell}\left(\hat{q}_{L}\right)\right)= \begin{cases}\frac{\left\langle\hat{q}_{L}, f_{\ell}\right\rangle^{2}}{2 \sigma^{2}\left\langle f_{\ell}, f_{\ell}\right\rangle} & \left\langle\hat{q}_{L}, f_{\ell}\right\rangle>0 \\ 0 & \text { Otherwise }\end{cases}
$$

A threshold value, $\lambda$, for $\Lambda_{\ell}$ must be set. If $\Lambda_{\ell}$ exceeds this value then the null hypothesis, $\mathcal{H}_{\emptyset}$, is rejected. The threshold is generally calculated using the probability of rejecting the null hypothesis when it is true, i.e. $\mathbb{P}\left[\Lambda_{\ell}\left(\hat{q}_{L}\right)>\lambda\right] \triangleq \alpha$, where $\mathbb{P}$ is the probability operator. The probability $\alpha$ is called the level of the test and is usually set to a value in the range of $0.1 \% \leqslant \alpha \leqslant 5 \%$. In the case of the pressurization and injection system, however, the severity of the modelling errors causes the stationarity and zero-mean assumptions on the disturbance $e(n)$ to fall. This makes it necessary to use very low levels of the test to prevent a high rate of false alarms. For this reason, no fault probability is chosen but instead the threshold $\lambda$ is chosen on the basis of experimental data.

\subsection{Laguerre analysis}

In order to decrease the extensive calculations implied by the GLR method, the analysis is carried out in the Laguerre domain. Laguerre coefficients (i.e. Laguerre spectrum) of a signal present a parsimonious way of describing its dynamic properties, when the signal exhibits a welldamped behaviour. $[7]$

The discrete time Laguerre functions in z-domain are

$$
L_{k}(z)=\frac{z \sqrt{1-a^{2}}}{z-a}\left(\frac{1-a z}{z-a}\right)^{k} k \geq 0,-1<a<1
$$

and constitute an orthonormal basis in $H^{2}\left(D^{c}\right)$ where $D^{c}$ is the complement of the unit disc in the complex plane. Orthonormality is understood in the sense of the usual inner product in $l^{2}\left(R_{+}\right)$which is, for two real functions $v$ and $w$ represented by their corresponding z-transforms $V(z)$ and $W(z)$, defined as

$$
\langle v, w\rangle=\frac{1}{2 \pi \jmath} \oint_{\Gamma} V(z) \overline{W(z)} \frac{d z}{z}
$$

where the contour $\Gamma$ represents the unit circle and - denotes complex conjugate. The time-domain discrete Laguerre functions can be obtained via inverse z-transform, thus $l_{k}(n)=\mathcal{Z}^{-1}\left\{L_{k}(z)\right\}$. The Laguerre coefficients of a function $f$ are equal to $f_{k}=\left\langle f, l_{k}\right\rangle$. Then the Laguerre spectrum of $f$ is defined as the set of $f_{k}, k=0, \ldots, \infty$.

It is straightforward to show that the scalar product of two functions $f$ and $g$ is equal to the scalar product of the Laguerre coefficients of these functions, i.e.

$$
\langle f, g\rangle=\sum_{k \geq 0} f_{k} g_{k}
$$

Thus, approximate values of leakage size and logarithmic GLR can be obtained by replacing the scalar products by the scalar products of the truncated Laguerre series of the leakage functions.

$$
\langle f, g\rangle \simeq \sum_{k=0}^{K-1} f_{k} g_{k}
$$

In order to obtain fast convergence of the Laguerre series of a function $f$, the parameter $a$ should be chosen so that $1 / a$ is close to the dominating time constant of $f$ [7]. The leakage functions $f_{\ell}$ are finite in time, and therefore $1 / a$ is chosen to be of the same order of magnitude as the time interval of the logged data set.

The quality of the approximations depends greatly on the number of Laguerre coefficients $K$. In this particular case, three coefficients suffice to get good results (see section 6).

Note that since the leakage functions look similar from one process cycle to another, the Laguerre-coefficients of these function can be calculated in advance, thus reducing the computational burden on the fault isolation system.

\subsection{Modified Laguerre spectrum}

Laguerre spectrum analysis is best suited for smoothly decaying signals. Since the finite-time leakage functions do not have this property, it is possible that a biased version of them could be approximated better by their truncated Laguerre spectrum. Define the biased finite-time signal $f$ as

$$
\bar{f}(n) \triangleq \begin{cases}f(n)-m_{f} & 0 \leq n \leq N \\ 0 & \text { Otherwise }\end{cases}
$$

where $m_{f}$ is an arbitrary number, for example the mean value or the final value of the finite-time function $f(n)$. It can be shown that the scalar product of two functions $f$ 
and $g$ can be expressed in the Laguerre coefficients of $\bar{f}$ and $\bar{g}$ as

$$
\langle f, g\rangle \simeq \sum_{k=0}^{K-1} \bar{f}_{k} \bar{g}_{k}+m_{g} \sum_{k=0}^{K-1} \bar{f}_{k} s_{k}+m_{f} \sum_{k=0}^{K-1} \bar{g}_{k} s_{k}+N m_{f} m_{g}
$$

where equality holds when $K \rightarrow \infty$. The constant $s_{k}$ is the sum of Laguerre function number $k$ over the time interval of $f$, thus

$$
s_{k} \triangleq \sum_{n=0}^{N-1} l_{k}(n)
$$

The scalar product can also be expressed in the Laguerre coefficients of the original functions $f$ and $g$ as

$$
\langle f, g\rangle \simeq \sum_{k=0}^{K-1} f_{k} g_{k}+m_{f} m_{g}\left(N-\sum_{k=0}^{K-1} s_{k}^{2}\right)
$$

Note that it can be shown that $\lim _{K \rightarrow \infty} \sum_{k=0}^{K-1} s_{k}^{2}=N$ and thus (6) approaches (5) as $K \rightarrow \infty$.

Since both the Laguerre coefficients of the leakage functions and also the sums of the Laguerre functions $s_{k}$ can be pre-computed, (6) gives a computationally efficient way of approximating the scalar products of (4), provided that the number of Laguerre coefficients is small.

\section{Implementation}

Leakage detection is a part of SafePCI, a PC-based system for control and monitoring of a pulverized coal injection plant. Since model-based control and leakage detection result in rather complex mathematical computations, the two tasks are divided and implemented in separate computers. An obvious advantage of this solution is an increased reliability. Fig. 2 shows the principal structure of SafePCI.

The system hardware is connected to the coal injection plant via buffer amplifiers. Altogether 40 signals are sampled and 4 actuators manipulated.

The leakage detection system is implemented as a computer program named PCIguard. PCIguard has the following features:

- On-line gas leakage detection

- Sampling and storing of all necessary signals for control and leakage detection.

- On-line plotting of measured signals

- Messaging in case of detected leakages, by sending facsimile transmissions to the responsible personal.

- Elementary process supervision in case of signals loss etc.

\section{Experiments}

The experiments have been performed at SSAB Tunnplåt, Luleå. Different leakages were artificially created during a period of six process cycles.

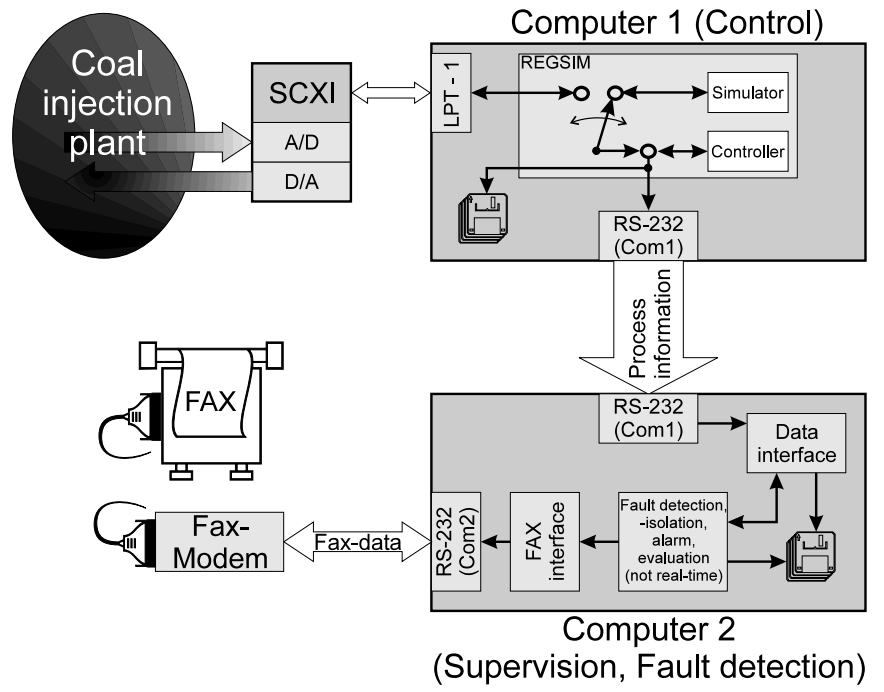

Figure 2: Hardware structure, data flow and used components

The logarithmic GLR, $\ln \left(\Lambda_{\ell}\left(\hat{q}_{L}\right)\right)$, was calculated with (4) using (6) as an approximation of the scalar products. The approximation was done with $K=3$ and the mean of the signals as the bias. The resulting logarithmic GLR for each leakage type and each cycle is shown as diagrams in Fig. 3 and Fig. 4. The dashed line in Fig. 4 shows the threshold for GLR when $\alpha=1 \%$ while the two dashed lines in Fig. 3 mark an interval for the threshold. Note that the hypothesis of leakage from the nitrogen net is absent in Fig. 3. The reason for this is that the pressure control valve, which connects the nitrogen net with the vessel, is open during pressurization and injection. There can, for obvious reasons, not be a leakage through an open valve.

A Leakage to the atmosphere was created during pressurization and injection for the first two process cycles. Thus, the right conclusions are drawn if the threshold for the logarithmic GLR is placed between the two dashed lines of Fig. 3. For the ventilation phase, leakage to the atmosphere was created during the first two process cycles and leakage from the nitrogen net during cycles 2-4. Fig. 4 shows that with level $1 \%$, the right conclusions are drawn for all process cycles except the second one, where there were two simultaneous leakages that nearly cancelled each other.

Fig. 5 shows a comparison of the results from pressurization and injection during the first process cycle (with leakage from the atmosphere) obtained with different numbers of Laguerre coefficients. Note that first three coefficients are sufficient to separate the two leakage hypotheses. In Fig. 6 an example of a leakage function with different Laguerre approximations is shown. Although the fit is significantly improved when the mean is removed, still no curve is a very good approximation. Evidently, in this case, the time-domain appearance is of limited importance compared to the Laguerre-domain. 


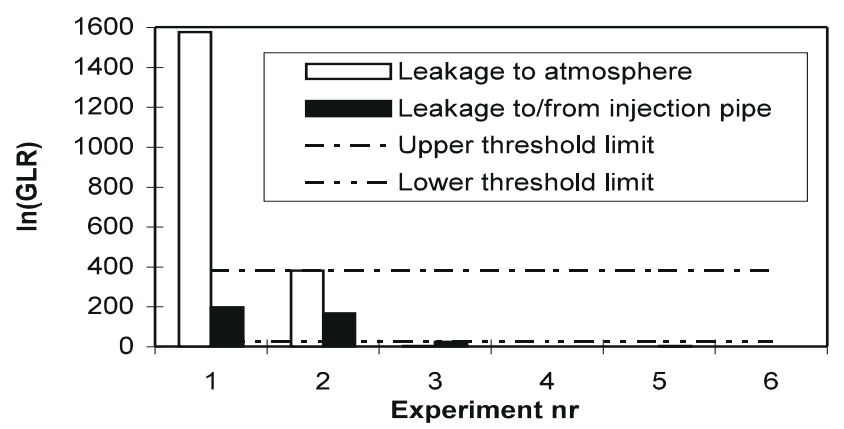

Figure 3: The logarithm of the GLR for each leakage type and each experiment during pressurization and injection

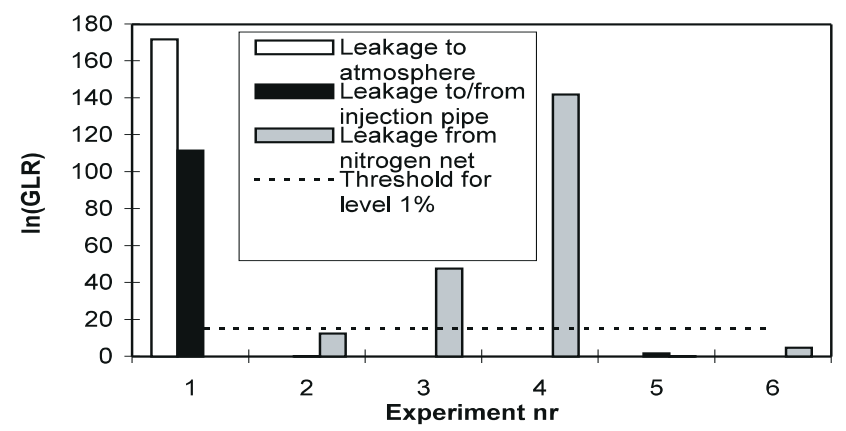

Figure 4: The logarithm of the GLR for each leakage type and each experiment during ventilation

\section{Conclusions}

Modelling and gas leakage detection in a pressurized system are discussed. It is shown that by use of modelbased methods, leakages can be detected and isolated with high accuracy. Furthermore, representing signals by their Laguerre coefficients is a means of reducing necessary calculations, without significantly affecting the accuracy of the results.

\section{Acknowledgment}

The authors want to thank SSAB Tunnplåt in Luleå, for making the coal injection plant and maintenance personnel available to us. Particularly, special thanks to Ingemar Lundström and Robert Johansson who helped with the experiments. Financial support of the Center for Process and System Automation (ProSA) at Luleå University of Technology provided by Norrbottens Forskningsråd is also gratefully acknowledged.

\section{References}

[1] W. Birk and A. Medvedev, "Pressure and flow control of a pulverized coal injection vessel," in Proceedings of the 1997 IEEE International Conference on Control Applications, pp. 127-132, 1997.

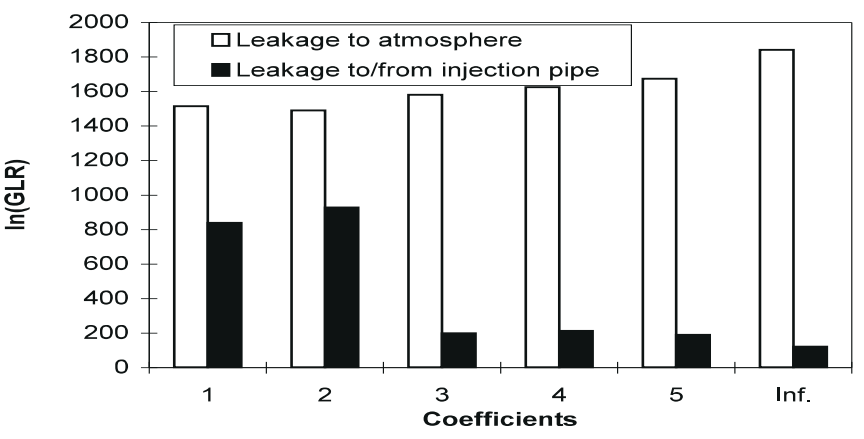

Figure 5: Comparison of the logarithmic GLR for different numbers of Laguerre coefficients

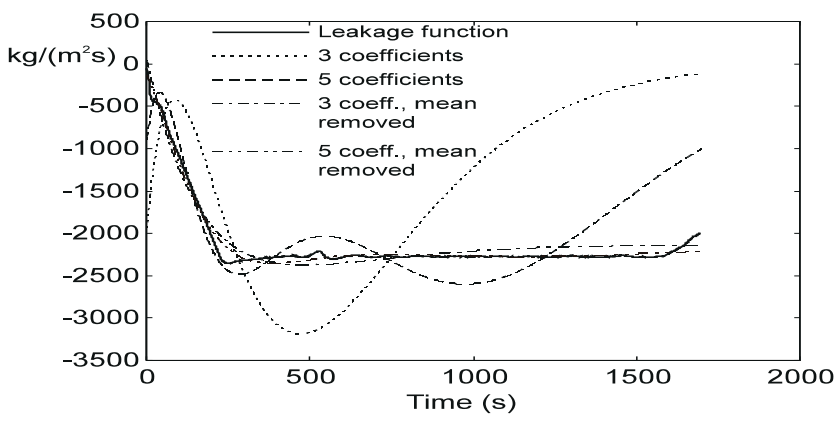

Figure 6: Leakage function for leakage to atmosphere during pressurization and injection compared to approximation with different numbers of Laguerre coefficients and with and without removing the mean

[2] P. M. Frank, "Fault diagnosis in dynamic systems using analytical and knowledge-based redundancy - a survey and some new results," Automatica, vol. 26, no. 3, pp. 459-474, 1990.

[3] E. A. Misawa and J. K. Hedrick, "Nonlinear observers - a state-of-the-art survey," Journal of Dynamic Systems, Measurment and Control, vol. 111, pp. 344-352, September 1989.

[4] J. Wünnenberg and P. M. Frank, "Dynamic model based incipient fault detection concepts for robots," in Preprints of the 11th IFAC World Congress, vol. 3, pp. 7681, 1990.

[5] A. Zhirabok and O. Preobrazhenskaya, "Robust fault detection and isolation in nonlinear systems," in Proceedings of IFAC Symposium "Safeprocess'94", pp. 244248, 1994.

[6] A. Johansson and A. Medvedev, "Model based leakage detection in a pulverized coal injection vessel." To be presented at the American Control Conference 1998.

[7] B. Wahlberg, "System identification using Laguerre models," IEEE Transaction on Automatic Control, vol. 36, pp. 551-562, May 1991. 